\title{
On the oscillation of fourth-order delay differential equations
}

Said R. Grace1, Jozef Džurina², Irena Jadlovská and Tongxing Li $i^{*}$

"Correspondence:

litongx2007@163.com

${ }^{3}$ School of Control Science and Engineering, Shandong University, Jinan, P.R. China

Full list of author information is

available at the end of the article

\section{Abstract}

In the paper, fourth-order delay differential equations of the form

$$
\left(r_{3}\left(r_{2}\left(r_{1} y^{\prime}\right)^{\prime}\right)^{\prime}\right)^{\prime}(t)+q(t) y(\tau(t))=0
$$

under the assumption

$$
\int_{t_{0}}^{\infty} \frac{\mathrm{d} t}{r_{i}(t)}<\infty, \quad i=1,2,3
$$

are investigated. Our newly proposed approach allows us to greatly reduce a number of conditions ensuring that all solutions of the studied equation oscillate. An example is also presented to test the strength and applicability of the results obtained.

MSC: $34 \mathrm{C} 10 ; 34 \mathrm{~K} 11$

Keywords: Linear differential equation; Delay; Fourth-order; Noncanonical operator; Oscillation

\section{Introduction}

Consider the fourth-order linear delay differential equation

$$
L_{4} y(t)+q(t) y(\tau(t))=0, \quad t \geq t_{0}>0,
$$

where

$$
L_{0} y=y, \quad L_{i} y=r_{i}(t)\left(L_{i-1} y\right)^{\prime}, \quad i=1,2,3, \quad L_{4} y=\left(L_{3} y\right)^{\prime} .
$$

In the sequel, we will assume that:

$\left(\mathrm{H}_{1}\right) r_{i} \in \mathcal{C}\left(\left[t_{0}, \infty\right), \mathbb{R}\right), i=1,2,3$ are positive and satisfy

$$
\pi_{i}\left(t_{0}\right):=\int_{t_{0}}^{\infty} \frac{\mathrm{d} t}{r_{i}(t)}<\infty ;
$$

$\left(\mathrm{H}_{2}\right) q \in \mathcal{C}\left(\left[t_{0}, \infty\right), \mathbb{R}\right)$ is nonnegative and does not vanish eventually;

$\left(\mathrm{H}_{3}\right) \tau \in \mathcal{C}^{1}\left(\left[t_{0}, \infty\right), \mathbb{R}\right), \tau(t) \leq t$, and $\lim _{t \rightarrow \infty} \tau(t)=\infty$.

c) The Author(s) 2019. This article is distributed under the terms of the Creative Commons Attribution 4.0 International License (http://creativecommons.org/licenses/by/4.0/), which permits unrestricted use, distribution, and reproduction in any medium, provided you give appropriate credit to the original author(s) and the source, provide a link to the Creative Commons license, and indicate if changes were made. 
Under a solution of (1.1), we mean a nontrivial function $y \in \mathcal{C}^{1}\left(\left[T_{y}, \infty\right), \mathbb{R}\right)$ with $T_{y} \geq t_{0}$, which has the property $L_{i} y \in \mathcal{C}^{1}\left(\left[T_{y}, \infty\right), \mathbb{R}\right)$ for $i=1,2,3$ and satisfies $(1.1)$ on $\left[T_{y}, \infty\right)$. We restrict our attention to those solutions of (1.1) which exist on some half-line $\left[T_{y}, \infty\right)$ and satisfy the condition

$$
\sup \{|y(t)|: T \leq t<\infty\}>0 \quad \text { for all } T \geq T_{y} .
$$

A solution $y$ of (1.1) is said to be oscillatory if it is neither eventually positive nor eventually negative. Otherwise, it is said to be nonoscillatory. The equation itself is termed oscillatory if all its solutions oscillate.

The foundations of vibration theory for continuous media established in the first half of the 18th century by the two close collaborators Daniel Bernoulli and Leonard Euler have generated the investigation of linear fourth-order differential equations [23]. Since then, the Euler-Bernoulli beam theory has shown to be of great practical importance due to its wide applications in civil, mechanical and aeronautical engineering and has been outlined in the literature over the years.

Being aware of the continuous interest in the study of self-excited oscillation phenomena which occur in bridges, it is worth mentioning that an oversimplified model concerns traveling waves in a suspension bridges $[8,18,19]$. Here, beams are used as the basis of supporting of the bridge or as the main-frame foundation in axles. The governing equation reads

$$
\frac{\partial^{2} u}{\partial t^{2}}+\delta \frac{\partial u}{\partial t}+\gamma \frac{\partial^{4} u}{\partial x^{4}}=0
$$

where $x$ is the coordinate along the beam axis, $t$ is the time, $u=u(x, t)$ is the lateral displacement, $\delta$ is the viscous damping coefficient and $\gamma$ is the stiffness coefficient per unit length. To find the traveling wave solutions of this partial differential equation, we may use the substitution of the form

$$
u(x, t)=w(s), \quad s=x-c t,
$$

with period $c$ and one has to solve the nonlinear fourth-order differential equation of the form

$$
\gamma w^{\prime \prime \prime \prime}(s)+c^{2} w^{\prime \prime}(s)+\delta c w^{\prime}(s)+f(w(s))=0 .
$$

As another important example on use of fourth-order equations, we mention the famous Swift-Hohenberg equation

$$
y^{(4)}(t)+k y^{\prime \prime}(t)+r(t) f(y(t))=0, \quad k>0,
$$

which serves as a model of pattern formation in many physical, chemical or biological systems [15].

It is also worth to mention the oscillatory muscle movement model represented by a fourth-order delay differential equation, which can arise due to the interaction of a muscle 
with its inertial load [20]. An unexpected area where fourth-order differential equations have occurred is in the context of number theory [4].

Because of the above motivating factors for the study of fourth-order differential equations, as well as because of the theoretical interest in generalizing and extending some known results from those given for lower-order equations, the study of oscillation of such equations has received considerable portion of attention. For a systematic summary of the most significant efforts made as regards this theory, the reader is referred to the monographs of Elias [7], Kiguradze and Chanturia [13], and Swanson [21].

As far as the oscillation theory of fourth-order differential equations is concerned, the problem of investigating ways of factoring disconjugate operator $L_{4} y$ has been of special interest.

Motivated by the famous work of George Pólya, Trench [22] showed that we can always write the operator $L_{4} y$ in an equivalent canonical form

$$
L_{4} y(t) \equiv \tilde{L}_{4} y(t)=p_{4}(t)\left(p_{3}\left(p_{2}\left(p_{1}\left(p_{0} y\right)^{\prime}\right)^{\prime}\right)^{\prime}\right)^{\prime}(t)
$$

such that the functions $p_{i} \in \mathcal{C}\left(\left[t_{0}, \infty\right), \mathbb{R}\right), i=0,1,2,3,4$ are positive,

$$
\int_{t_{0}}^{\infty} \frac{\mathrm{d} s}{p_{i}(s)}=\infty, \quad i=1,2,3
$$

and uniquely determined up to positive multiplicative constants with the product 1 . The explicit forms of the functions $p_{i}$ generally depend on whether the integrals $\pi_{i}(i=1,2,3)$, which we defined in $\left(\mathrm{H}_{1}\right)$, are convergent or divergent. Consequently, the investigation of the qualitative behavior of canonical fourth-order functional differential equations of the form

$$
\tilde{L}_{4} y(t)+q(t) y(\tau(t))=0
$$

its generalizations or particular cases, especially with regard to their oscillatory properties, has become the subject of intensive research; see, for instance, $[1-3,5,11,12,16,17,24-$ $26]$ and the references cited therein.

The main advantage of studying (1.1) in canonical form (1.2) essentially lies in the direct application of the well-known Kiguradze lemma [13, Lemma 1], which allows one to classify the set of possible nonoscillatory solutions. In particular, if $y$ is a positive solution of the canonical equation (1.2), then there are only two possible cases for $y$ :

$$
\begin{array}{lllll}
y>0, & \tilde{L}_{1} y>0, & \tilde{L}_{2} y<0, & \tilde{L}_{3} y>0, & \tilde{L}_{4} y<0, \\
y>0, & \tilde{L}_{1} y>0, & \tilde{L}_{2} y>0, & \tilde{L}_{3} y>0, & \tilde{L}_{4} y<0,
\end{array}
$$

for $t$ large enough.

However, the formulas for the corresponding functions $p_{i}$ resulting from Trench's theory of canonical operators are in general too complicated to allow the practical application of existing results obtained for canonical equations. Another possible approach elaborated by several authors is to investigate the original equation, at the cost of the existence of 
additional classes of possible nonoscillatory solutions. In particular, the authors in $[9,10]$ established oscillation results for (1.1) under assumptions

$$
r_{1}=r_{2}=1 \text { and } \pi_{3}\left(t_{0}\right)<\infty
$$

and

$$
r_{1}=r_{3}=1 \text { and } \pi_{2}\left(t_{0}\right)<\infty
$$

respectively. Although the technique used in these papers is different, their results have in common that the oscillation of the studied equation was ensured via four independent conditions, eliminating nonoscillatory solutions pertaining to particular classes.

Very recently, Džurina and Jadlovská [6] investigated the oscillatory behavior of thirdorder differential equations of the form

$$
L_{3} y(t)+q(t) y(\tau(t))=0
$$

under the condition

$$
\pi_{i}\left(t_{0}\right)<\infty, \quad i=1,2
$$

By careful observation, they pointed out that various conditions, traditionally imposed in the existing results are redundant. This observation led to the gain of various twocondition oscillation criteria for (1.3).

To the best of our knowledge, there is nothing known about the oscillation of (1.1) under the assumption $\left(\mathrm{H}_{1}\right)$. Inspired by the ideas adopted in [6], our primary goal is to fill this gap by presenting simple criteria for the oscillation of all solutions of (1.1). Most importantly, we stress that the nonexistence of eight possible classes of nonoscillatory solutions (see Lemma 1 ) is shown only through two conditions. Our newly proposed approach could hopefully serve as a reference in the less-developed theory for noncanonical equations. Finally, we illustrate the importance of the results obtained via Euler-type equations.

\section{Main results}

For the sake of clarity, we list the functions to be used in the paper. That is, for $t \geq t_{*} \geq t_{0}$, we put

$$
\begin{aligned}
& \pi_{12}(t)=\int_{t}^{\infty} \frac{\pi_{2}(s)}{r_{1}(s)} \mathrm{d} s, \quad \pi_{23}(t)=\int_{t}^{\infty} \frac{\pi_{3}(s)}{r_{2}(s)} \mathrm{d} s, \quad \pi_{123}(t)=\int_{t}^{\infty} \frac{\pi_{23}(s)}{r_{1}(s)} \mathrm{d} s, \\
& Q\left(t, t_{*}\right)=\int_{t_{*}}^{t} \frac{1}{r_{2}(v)} \int_{t_{*}}^{v} \frac{1}{r_{3}(u)} \int_{t_{*}}^{u} q(s) \mathrm{d} s \mathrm{~d} u \mathrm{~d} v, \quad \tilde{Q}\left(t, t_{*}\right)=\int_{t_{*}}^{t} \frac{q(s) \pi_{123}(\tau(s))}{\pi_{3}(\tau(s))} \mathrm{d} s .
\end{aligned}
$$

As usual, all functional inequalities considered in this paper are supposed to be satisfied for all $t$ large enough. In what follows, we need only to consider eventually positive solutions of (1.1), since if $y$ satisfies (1.1), then so does $-y$.

Lemma 1 Assume that $\left(\mathrm{H}_{1}\right)-\left(\mathrm{H}_{3}\right)$ hold and $y$ is an eventually positive solution to (1.1). Then there exists a $t_{1} \in\left[t_{0}, \infty\right)$ such that $y$ satisfies one of the following cases:

$$
\text { case }(1): \quad y>0, \quad L_{1} y>0, \quad L_{2} y>0, \quad L_{3} y>0, \quad L_{4} y \leq 0 \text {, }
$$




$\begin{array}{lllll}\text { case }(2): \quad y>0, & L_{1} y>0, & L_{2} y>0, & L_{3} y<0, & L_{4} y \leq 0, \\ \text { case (3): } y>0, & L_{1} y>0, & L_{2} y<0, & L_{3} y>0, & L_{4} y \leq 0, \\ \text { case (4): } y>0, & L_{1} y>0, & L_{2} y<0, & L_{3} y<0, & L_{4} y \leq 0, \\ \text { case (5): } y>0, & L_{1} y<0, & L_{2} y>0, & L_{3} y>0, & L_{4} y \leq 0, \\ \text { case (6): } y>0, & L_{1} y<0, & L_{2} y>0, & L_{3} y<0, & L_{4} y \leq 0, \\ \text { case (7): } y>0, & L_{1} y<0, & L_{2} y<0, & L_{3} y>0, & L_{4} y \leq 0, \\ \text { case (8): } y>0, & L_{1} y<0, & L_{2} y<0, & L_{3} y<0, & L_{4} y \leq 0,\end{array}$

for $t \geq t_{1}$.

Proof The proof is obvious and so we omit it.

We start with a simple condition ensuring the nonexistence of solutions of types (1)(4). As will be shown later, this condition is already included in those eliminating positive decreasing solutions.

Lemma 2 Assume that $\left(\mathrm{H}_{1}\right)-\left(\mathrm{H}_{3}\right)$ hold. Let y be an eventually positive solution of (1.1). If

$$
Q\left(\infty, t_{0}\right)=\infty
$$

then cases (1)-(4) from Lemma 1 are impossible.

Proof First of all, it is important to note that if both $\left(\mathrm{H}_{1}\right)$ and $(2.1)$ hold, then

$$
\int_{t_{0}}^{\infty} \frac{1}{r_{3}(u)} \int_{t_{0}}^{u} q(s) \mathrm{d} s \mathrm{~d} u=\int_{t_{0}}^{\infty} q(s) \mathrm{d} s=\infty
$$

Now, assume on the contrary that $y(t)$ is an eventually positive solution of (1.1) satisfying one of the cases (1)-(4) from Lemma 1 and pick a $t_{1} \in\left[t_{0}, \infty\right)$ such that $y(\tau(t))>0$ for $t \geq t_{1}$. Since $y$ is increasing, there exist a constant $c>0$ and a $t_{2} \geq t_{1}$ such that $y(\tau(t)) \geq c$ for $t \geq t_{2}$. Using this inequality in (1.1), we get

$$
-L_{4} y(t) \geq c q(t) \quad \text { for } t \geq t_{2}
$$

Integrating (2.3) from $t_{2}$ to $t$, we find

$$
-L_{3} y(t)+L_{3} y\left(t_{2}\right) \geq c \int_{t_{2}}^{t} q(s) \mathrm{d} s
$$

If we assume that $y$ belongs either to case (1) or case (3), then from (2.2) and (2.4), we obtain

$$
L_{3} y\left(t_{2}\right) \geq c \int_{t_{2}}^{t} q(s) \mathrm{d} s \rightarrow \infty \quad \text { as } t \rightarrow \infty,
$$

which contradicts the fact that $L_{3} y$ is nonincreasing. 
Next, assume that case (2) holds. Then (2.4) becomes

$$
-L_{3} y(t) \geq c \int_{t_{2}}^{t} q(s) \mathrm{d} s
$$

that is,

$$
-\left(L_{2} y\right)^{\prime}(t) \geq \frac{c}{r_{3}(t)} \int_{t_{2}}^{t} q(s) \mathrm{d} s
$$

Integrating (2.6) from $t_{2}$ to $t$, we have

$$
L_{2} y\left(t_{2}\right)-L_{2} y(t) \geq c \int_{t_{2}}^{t} \frac{1}{r_{3}(u)} \int_{t_{2}}^{u} q(s) \mathrm{d} s \mathrm{~d} u
$$

which, by virtue of (2.2), gives

$$
L_{2} y\left(t_{2}\right) \geq c \int_{t_{2}}^{t} \frac{1}{r_{3}(u)} \int_{t_{2}}^{u} q(s) \mathrm{d} s \mathrm{~d} u \rightarrow \infty \quad \text { as } t \rightarrow \infty,
$$

which clearly contradicts the fact that $L_{2} y$ is decreasing.

Finally, let us assume that case (4) holds. In the same way as in the previous case, we arrive at (2.7), namely,

$$
-\left(L_{1} y\right)^{\prime}(t) \geq \frac{c}{r_{2}(t)} \int_{t_{2}}^{t} \frac{1}{r_{3}(u)} \int_{t_{2}}^{u} q(s) \mathrm{d} s \mathrm{~d} u
$$

Integrating this inequality from $t_{2}$ to $t$, we get

$$
L_{1} y\left(t_{2}\right)-L_{1} y(t) \geq c \int_{t_{2}}^{t} \frac{1}{r_{2}(v)} \int_{t_{2}}^{v} \frac{1}{r_{3}(u)} \int_{t_{2}}^{u} q(s) \mathrm{d} s \mathrm{~d} u \mathrm{~d} v=c Q\left(t, t_{2}\right),
$$

which in view of $(2.1)$ yields

$$
L_{1} y\left(t_{2}\right) \geq c Q\left(t, t_{2}\right) \rightarrow \infty \quad \text { as } t \rightarrow \infty
$$

which contradicts the fact that $L_{1} y$ is decreasing.

The proof is complete.

In the following result, a simple condition ensuring that any nonoscillatory solution converges to zero as $t \rightarrow \infty$ is established.

Theorem 1 Assume that $\left(\mathrm{H}_{1}\right)-\left(\mathrm{H}_{3}\right)$ hold. If

$$
\int_{t_{0}}^{\infty} \frac{Q\left(t, t_{0}\right)}{r_{1}(t)} \mathrm{d} t=\infty
$$

then any solution $y$ of $(1.1)$ is oscillatory or $\lim _{t \rightarrow \infty} y(t)=0$. 
Proof Assume that $y$ is a nonoscillatory solution of $(1.1)$ on $\left[t_{0}, \infty\right)$. Without loss of generality, we may take a $t_{1} \geq t_{0}$ such that $y(t)>0$ and $y(\tau(t))>0$ for $t \geq t_{1}$. By Lemma 1 , eight possible cases may occur for $t \geq t_{1}$.

Since (2.10) together with $\left(\mathrm{H}_{1}\right)$ implies that $\int_{t_{0}}^{\infty} Q\left(t, t_{0}\right) \mathrm{d} t$ cannot be bounded, by Lemma 2, cases (1)-(4) are impossible.

Let one of the cases (5)-(8) hold. Since $y$ is decreasing, there exists a finite nonnegative limit $y(\infty)=\lim _{t \rightarrow \infty} y(t)=c$. Assume on the contrary that $c>0$. Then there exists a $t_{2} \geq$ $t_{1}$ such that $y(\tau(t)) \geq c$ for $t \geq t_{2}$ and inequality (2.3) is satisfied. Then one can arrive at contradiction (2.5) in cases (5) and (7), and contradiction (2.8) in case (6). Thus, we conclude that $c=0$.

If we assume that case (8) holds, then we get (2.9), that is,

$$
-L_{1} y(t) \geq c Q\left(t, t_{2}\right)
$$

or

$$
-y^{\prime}(t) \geq \frac{c}{r_{1}(t)} Q\left(t, t_{2}\right)
$$

Integrating the above inequality from $t_{2}$ to $t$, we obtain

$$
y\left(t_{2}\right) \geq c \int_{t_{2}}^{t} \frac{Q\left(s, t_{2}\right)}{r_{1}(s)} \mathrm{d} s .
$$

However, the integral on the right-hand side of the above inequality tends to $\infty$ as $t \rightarrow \infty$ due to (2.10), which contradicts the fact that $y$ is decreasing.

The proof is complete.

In the sequel, we present various two-condition oscillation criteria for (1.1).

Theorem 2 Assume that $\left(\mathrm{H}_{1}\right)-\left(\mathrm{H}_{3}\right)$ hold and $\tau$ is nondecreasing. If

$$
\limsup _{t \rightarrow \infty} A\left(t, t_{1}\right)>1
$$

for any $t_{1} \geq t_{0}$, where

$$
A\left(t, t_{1}\right):=\min \left\{\pi_{1}(t) Q\left(t, t_{1}\right), \pi_{3}(t) \tilde{Q}\left(t, t_{1}\right)\right\},
$$

then (1.1) is oscillatory.

Proof Assume that $y$ is a nonoscillatory solution of $(1.1)$ on $\left[t_{0}, \infty\right)$. Without loss of generality, we may take a $t_{1} \geq t_{0}$ such that $y(t)>0$ and $y(\tau(t))>0$ for $t \geq t_{1}$. By Lemma 1 , eight possible cases may occur for $t \geq t_{1}$.

At first, it is useful to note that, in view of $\left(\mathrm{H}_{1}\right)$, it is necessary for the validity of $(2.11)$ that

$$
Q\left(\infty, t_{0}\right)=\tilde{Q}\left(\infty, t_{0}\right)=\infty
$$

From Lemma 2, the above condition ensures that cases (1)-(4) from Lemma 1 are impossible. We shall consider the remaining possible cases (5)-(8) separately. 
Assume that case (5) holds. From the monotonicity of $L_{2} y$, we deduce that

$$
-L_{1} y(t) \geq L_{1} y(\infty)-L_{1} y(t)=\int_{t}^{\infty} \frac{1}{r_{2}(s)} L_{2} y(s) \mathrm{d} s \geq L_{2} y(t) \pi_{2}(t)
$$

that is,

$$
-y^{\prime}(t) \geq L_{2} y(t) \frac{\pi_{2}(t)}{r_{1}(t)}
$$

Integrating the above inequality from $t$ to $\infty$, we get

$$
y(t) \geq L_{2} y(t) \int_{t}^{\infty} \frac{\pi_{2}(s)}{r_{1}(s)} \mathrm{d} s=L_{2} y(t) \pi_{12}(t) .
$$

Using (2.13) and the increasing property of $L_{2} y$ in (1.1), there exist a constant $c>0$ and a $t_{2} \geq t_{1}$ such that

$$
-L_{4} y(t)=q(t) y(\tau(t)) \geq q(t) L_{2} y(\tau(t)) \pi_{12}(\tau(t)) \geq c q(t) \pi_{12}(\tau(t)) \quad \text { for } t \geq t_{2} .
$$

Integrating the above inequality from $t_{2}$ to $t$, we have

$$
L_{3} y\left(t_{2}\right) \geq L_{3} y(t)+c \int_{t_{2}}^{t} q(s) \pi_{12}(\tau(s)) \mathrm{d} s .
$$

Taking $\left(\mathrm{H}_{1}\right)$ and (2.12) into account, it is easy to see that

$$
\infty=\tilde{Q}\left(\infty, t_{0}\right)=\int_{t_{0}}^{\infty} \frac{q(s) \pi_{123}(\tau(s))}{\pi_{3}(\tau(s))} \mathrm{d} s \leq \int_{t_{0}}^{\infty} q(s) \pi_{12}(\tau(s)) \mathrm{d} s
$$

Using (2.15) in (2.14), we arrive at a contradiction with the fact that $L_{3} y$ is nonincreasing. Assume that case (6) holds. From the monotonicity of $L_{3} y$, we have

$$
L_{2} y(t)-L_{2} y(\infty)=-\int_{t}^{\infty} \frac{1}{r_{3}(s)} L_{3} y(s) \mathrm{d} s \geq-L_{3} y(t) \pi_{3}(t) .
$$

Therefore,

$$
\left(\frac{L_{2} y}{\pi_{3}}\right)^{\prime}(t)=\frac{L_{3} y(t) \pi_{3}(t)+L_{2} y(t)}{\pi_{3}^{2}(t) r_{3}(t)} \geq 0
$$

which implies that $L_{2} y / \pi_{3}$ is nondecreasing. Using further this property, we obtain

$$
-L_{1} y(t) \geq \int_{t}^{\infty} \frac{1}{r_{2}(s)} L_{2} y(s) \mathrm{d} s \geq \frac{L_{2} y(t)}{\pi_{3}(t)} \int_{t}^{\infty} \frac{\pi_{3}(s)}{r_{2}(s)} \mathrm{d} s=\frac{L_{2} y(t)}{\pi_{3}(t)} \pi_{23}(t) .
$$

Hence,

$$
\left(-\frac{L_{1} y}{\pi_{23}}\right)^{\prime}(t)=\frac{-L_{2} y(t) \pi_{23}(t)-L_{1} y(t) \pi_{3}(t)}{\pi_{23}^{2}(t) r_{2}(t)} \geq 0
$$


and so $-L_{1} y / \pi_{23}$ is nondecreasing. Finally, we arrive at

$$
y(t) \geq-\int_{t}^{\infty} \frac{1}{r_{1}(s)} L_{1} y(s) \mathrm{d} s \geq-\frac{L_{1} y(t)}{\pi_{23}(t)} \int_{t}^{\infty} \frac{\pi_{23}(s)}{r_{1}(s)} \mathrm{d} s=-\frac{L_{1} y(t)}{\pi_{23}(t)} \pi_{123}(t) .
$$

Using (2.17) in the above inequality, we get

$$
y(t) \geq \frac{L_{2} y(t)}{\pi_{3}(t)} \pi_{123}(t) .
$$

Therefore,

$$
-L_{4} y(t)=q(t) y(\tau(t)) \geq \frac{q(t) \pi_{123}(\tau(t))}{\pi_{3}(\tau(t))} L_{2} y(\tau(t))
$$

Integrating this inequality from $t_{1}$ to $t$ and using the monotonicity of $L_{2} y$, we find

$$
\begin{aligned}
-L_{3} y(t) & \geq \int_{t_{1}}^{t} \frac{q(s) \pi_{123}(\tau(s))}{\pi_{3}(\tau(s))} L_{2} y(\tau(s)) \mathrm{d} s \\
& \geq L_{2} y(\tau(t)) \int_{t_{1}}^{t} \frac{q(s) \pi_{123}(\tau(s))}{\pi_{3}(\tau(s))} \mathrm{d} s \geq L_{2} y(t) \tilde{Q}\left(t, t_{1}\right) .
\end{aligned}
$$

From (2.16) and (2.19), we obtain

$$
-L_{3} y(t) \geq-L_{3} y(t) \tilde{Q}\left(t, t_{1}\right) \pi_{3}(t)
$$

Dividing the above inequality by $-L_{3} y$ and taking the lim sup on both sides of the resulting inequality, one arrives at a contradiction with (2.11).

Assume that case (7) holds. From the decreasing property of $L_{1} y$, we get

$$
y(t) \geq y(t)-y(\infty)=-\int_{t}^{\infty} \frac{1}{r_{1}(s)} L_{1} y(s) \mathrm{d} s \geq-\pi_{1}(t) L_{1} y(t) .
$$

Thus,

$$
\left(\frac{y}{\pi_{1}}\right)^{\prime}(t)=\frac{L_{1} y(t) \pi_{1}(t)+y(t)}{\pi_{1}^{2}(t) r_{1}(t)} \geq 0
$$

which means that $y / \pi_{1}$ is nondecreasing. Integrating (1.1) from $t_{1}$ to $t$ and using the monotonicity of $y$, we conclude that

$$
L_{3} y\left(t_{1}\right)=L_{3} y(t)+\int_{t_{1}}^{t} q(s) y(\tau(s)) \mathrm{d} s \geq \frac{y\left(t_{1}\right)}{\pi_{1}\left(t_{1}\right)} \int_{t_{1}}^{t} q(s) \pi_{1}(s) \mathrm{d} s .
$$

On the other hand, using $\left(\mathrm{H}_{1}\right)$ and (2.15), it is easy to see that, for any constant $k>0$,

$$
\infty=\int_{t_{1}}^{\infty} q(s) \pi_{12}(s) \mathrm{d} s \leq k \int_{t_{1}}^{\infty} q(s) \pi_{1}(s) \mathrm{d} s .
$$

This in view of inequality (2.20) contradicts the fact that $L_{3} y$ is nonincreasing. 
Assume that case (8) holds. Integrating (1.1) from $t_{1}$ to $t$, we have

$$
-L_{3} y(t) \geq \int_{t_{1}}^{t} q(s) y(\tau(s)) \mathrm{d} s \geq y(\tau(t)) \int_{t_{1}}^{t} q(s) \mathrm{d} s .
$$

Dividing both sides of the above inequality by $r_{3}(t)$ and integrating the resulting inequality again from $t_{1}$ to $t$, we get

$$
-L_{2} y(t) \geq \int_{t_{1}}^{t} \frac{y(\tau(u))}{r_{3}(u)} \int_{t_{1}}^{u} q(s) \mathrm{d} s \mathrm{~d} u \geq y(\tau(t)) \int_{t_{1}}^{t} \frac{1}{r_{3}(u)} \int_{t_{1}}^{u} q(s) \mathrm{d} s \mathrm{~d} u .
$$

Similarly, we obtain

$$
\begin{aligned}
-L_{1} y(t) & \geq y(\tau(t)) \int_{t_{1}}^{t} \frac{1}{r_{2}(v)} \int_{t_{1}}^{v} \frac{1}{r_{3}(u)} \int_{t_{1}}^{u} q(s) \mathrm{d} s \mathrm{~d} u \mathrm{~d} v \\
& =y(\tau(t)) Q\left(t, t_{1}\right) \geq y(t) Q\left(t, t_{1}\right) \geq-L_{1} y(t) \pi_{1}(t) Q\left(t, t_{1}\right),
\end{aligned}
$$

that is,

$$
1 \geq \pi_{1}(t) Q\left(t, t_{1}\right)
$$

which clearly contradicts (2.11).

The proof is complete.

Theorem 3 Assume that $\left(\mathrm{H}_{1}\right)-\left(\mathrm{H}_{3}\right)$ hold and $\tau$ is nondecreasing. If

$$
\liminf _{t \rightarrow \infty} \int_{\tau(t)}^{t} B\left(s, t_{1}\right) \mathrm{d} s>\frac{1}{\mathrm{e}}
$$

for any $t_{1} \geq t_{0}$, where

$$
B\left(t, t_{1}\right):=\min \left\{\frac{Q\left(t, t_{1}\right)}{r_{1}(t)}, \frac{\tilde{Q}\left(t, t_{1}\right)}{r_{3}(t)}\right\}
$$

then (1.1) is oscillatory.

Proof Assume that $y$ is a nonoscillatory solution of (1.1) on $\left[t_{0}, \infty\right)$. Without loss of generality, we may take a $t_{1} \geq t_{0}$ such that $y(t)>0$ and $y(\tau(t))>0$ for $t \geq t_{1}$. By Lemma 1 , eight possible cases may occur for $t \geq t_{1}$.

First, note that it is necessary for the validity of (2.23) that

$$
\int_{t_{0}}^{\infty} B\left(t, t_{1}\right) \mathrm{d} t=\infty
$$

which in view of $\left(\mathrm{H}_{1}\right)$ implies that (2.12) holds. From Lemma 2, we see that the above condition ensures that cases (1)-(4) from Lemma 1 are impossible. We will consider the remaining possible cases (5)-(8) separately. 
Since cases (5) and (7) can be treated exactly as in the proof of Theorem 2, we omit this part of the proof. Assume that case (6) holds. Proceeding as in the proof of Theorem 2 case (6), we arrive at (2.19), i.e.,

$$
-L_{3} y(t) \geq L_{2} y(\tau(t)) \int_{t_{1}}^{t} \frac{q(s) \pi_{123}(\tau(s))}{\pi_{3}(\tau(s))} \mathrm{d} s,
$$

that is,

$$
x^{\prime}(t)+\frac{\tilde{Q}\left(t, t_{1}\right)}{r_{3}(t)} x(\tau(t)) \leq 0,
$$

where we set $x(t)=L_{2} y(t)>0$. It follows from (2.23) that

$$
\liminf _{t \rightarrow \infty} \int_{\tau(t)}^{t} \frac{\tilde{Q}\left(s, t_{1}\right)}{r_{3}(s)} \mathrm{d} s>\frac{1}{\mathrm{e}}
$$

however, by [14, Theorem 2.1.1], this condition ensures that inequality (2.24) does not possess a positive solution, which is a contradiction with our initial assumption.

Assume that case (8) holds. Proceeding as in the proof of Theorem 2 case (8), we arrive at (2.22), i.e.,

$$
-L_{1} y(t) \geq y(\tau(t)) Q\left(t, t_{1}\right)
$$

or

$$
y^{\prime}(t)+\frac{Q\left(t, t_{1}\right)}{r_{1}(t)} y(\tau(t)) \leq 0 .
$$

Similar to case (7), we arrive at a contradiction.

The proof is complete.

The last criterion is obtained by employing the classical Riccati transformation technique.

Theorem 4 Assume that $\left(\mathrm{H}_{1}\right)-\left(\mathrm{H}_{3}\right)$ hold. If, for all sufficiently large $t_{1} \geq t_{0}$,

$$
\limsup _{t \rightarrow \infty} \int_{t_{1}}^{t}\left(q(s) \pi_{123}(s)-\frac{\pi_{23}(s)}{4 r_{1}(s) \pi_{123}(s)}\right) \mathrm{d} s=\infty
$$

and

$$
\limsup _{t \rightarrow \infty} \int_{t_{1}}^{t}\left(\frac{\pi_{1}(v)}{r_{2}(v)} \int_{t_{1}}^{v} \frac{1}{r_{3}(u)} \int_{t_{1}}^{u} q(s) \mathrm{d} s \mathrm{~d} u-\frac{1}{4 \pi_{1}(v) r_{1}(v)}\right) \mathrm{d} v=\infty
$$

then (1.1) is oscillatory.

Proof Suppose for the sake of contradiction that $y$ is a nonoscillatory solution of (1.1) on $\left[t_{0}, \infty\right)$. Without loss of generality, we may take a $t_{1} \geq t_{0}$ such that $y(t)>0$ and $y(\tau(t))>0$ for $t \geq t_{1}$. By Lemma 1, eight possible cases may occur for $t \geq t_{1}$. From (2.27), we see that

$$
\int_{t_{0}}^{\infty} \frac{\pi_{1}(v)}{r_{2}(v)} \int_{t_{1}}^{v} \frac{1}{r_{3}(u)} \int_{t_{1}}^{u} q(s) \mathrm{d} s \mathrm{~d} u \mathrm{~d} v=\infty
$$


which in view of $\left(\mathrm{H}_{1}\right)$ implies that $Q\left(\infty, t_{0}\right)=\infty$. Thus, by Lemma 2 , cases $(1)-(4)$ from Lemma 1 are impossible. Therefore, it is enough to consider cases (5)-(8).

Assume that case (5) holds. From (2.26), we have

$$
\int_{t_{0}}^{\infty} q(s) \pi_{123}(s) \mathrm{d} s=\infty
$$

Then, proceeding as in the proof of Theorem 2 case (5), we arrive at the contradiction. Assume that case (6) holds. Let us define the function

$$
w(t)=\frac{L_{3} y(t)}{y(t)}<0
$$

Combining (2.16) and (2.18), we obtain

$$
y(t) \geq L_{3} y(t) \pi_{123}(t),
$$

which yields

$$
-1 \leq w(t) \pi_{123}(t)<0 .
$$

Also, proceeding as in the proof of Theorem 2 case (6), we derive from (2.16) and (2.17) that

$$
y^{\prime}(t) \geq-L_{3} y(t) \frac{\pi_{23}(t)}{r_{1}(t)}
$$

By (1.1), (2.29), and the monotonicity of $y$, we conclude that

$$
w^{\prime}(t)=\frac{L_{4} y(t)}{y(t)}-\frac{L_{3} y(t) y^{\prime}(t)}{y^{2}(t)} \leq-q(t) \frac{y(\tau(t))}{y(t)}-\frac{\left(L_{3} y(t)\right)^{2} \pi_{23}(t)}{r_{1}(t) y^{2}(t)} \leq-q(t)-w^{2}(t) \frac{\pi_{23}(t)}{r_{1}(t)} .
$$

Multiplying the above inequality by $\pi_{123}$ and integrating the resulting inequality from $t_{1}$ to $t$, we get

$$
\begin{aligned}
& w(t) \pi_{123}(t)-w\left(t_{1}\right) \pi_{123}\left(t_{1}\right)+\int_{t_{1}}^{t} w(s) \frac{\pi_{23}(s)}{r_{1}(s)} \mathrm{d} s+\int_{t_{1}}^{t} q(s) \pi_{123}(s) \mathrm{d} s \\
& +\int_{t_{1}}^{t} w^{2}(s) \frac{\pi_{23}(s) \pi_{123}(s)}{r_{1}(s)} \mathrm{d} s \leq 0 .
\end{aligned}
$$

Therefore, by virtue of (2.28),

$$
\int_{t_{1}}^{t}\left(q(s) \pi_{123}(s)-\frac{\pi_{23}(s)}{4 r_{1}(s) \pi_{123}(s)}\right) \mathrm{d} s \leq w\left(t_{1}\right) \pi_{123}\left(t_{1}\right)+1,
$$

which contradicts (2.26).

Assume that case (7) holds. Note that

$$
\int_{t_{0}}^{\infty} q(s) \pi_{123}(s) \mathrm{d} s=\infty
$$


is necessary for (2.26). Then, for any $k>0$, we have

$$
\infty=\int_{t_{0}}^{\infty} q(s) \pi_{123}(s) \mathrm{d} s \leq k \int_{t_{1}}^{\infty} \pi_{12}(s) \mathrm{d} s .
$$

Proceeding as in the proof of Theorem 2 case (7), we arrive at the contradiction.

Assume that case (8) holds. Let us define the function

$$
v(t)=\frac{L_{1} y(t)}{y(t)}<0
$$

From (2.21), we obtain

$$
-L_{2} y(t) \geq y(t) \int_{t_{1}}^{t} \frac{1}{r_{3}(u)} \int_{t_{1}}^{u} q(s) \mathrm{d} s \mathrm{~d} u .
$$

On the other hand, from (2.25), we see that

$$
-1 \leq v(t) \pi_{1}(t)<0 .
$$

Then

$$
v^{\prime}(t)=\frac{L_{2} y(t)}{r_{2}(t) y(t)}-\frac{\left(L_{1} y(t)\right)^{2}}{r_{1}(t) y^{2}(t)} \leq-\frac{1}{r_{2}(t)} \int_{t_{1}}^{t} \frac{1}{r_{3}(u)} \int_{t_{1}}^{u} q(s) \mathrm{d} s \mathrm{~d} u-\frac{v^{2}(t)}{r_{1}(t)} .
$$

Now, multiplying both sides of the above inequality by $\pi_{1}(t)$ and integrating the resulting inequality from $t_{1}$ to $t$, we get

$$
\begin{aligned}
& v(t) \pi_{1}(t)-v\left(t_{1}\right) \pi_{1}\left(t_{1}\right)+\int_{t_{1}}^{t} \frac{v(s)}{r_{1}(s)} \mathrm{d} s+\int_{t_{1}}^{t} \frac{\pi_{1}(x)}{r_{2}(x)} \int_{t_{1}}^{x} \frac{1}{r_{3}(u)} \int_{t_{1}}^{u} q(s) \mathrm{d} s \mathrm{~d} u \mathrm{~d} x \\
& +\int_{t_{1}}^{t} v^{2}(s) \frac{\pi_{1}(s)}{r_{1}(s)} \mathrm{d} s \leq 0 .
\end{aligned}
$$

Hence, in view of (2.30),

$$
\int_{t_{1}}^{t}\left(\frac{\pi_{1}(x)}{r_{2}(x)} \int_{t_{1}}^{x} \frac{1}{r_{3}(u)} \int_{t_{1}}^{u} q(s) \mathrm{d} s \mathrm{~d} u-\frac{1}{4 \pi_{1}(x) r_{1}(x)}\right) \mathrm{d} x \leq v\left(t_{1}\right) \pi_{1}\left(t_{1}\right)+1,
$$

which contradicts (2.27).

The proof is complete.

We conclude the paper by providing an example that illustrates the applicability and strength of the results obtained.

Example 1 Let us consider the fourth-order differential equation of Euler type

$$
\left(t^{2}\left(t^{2}\left(t^{2} y^{\prime}(t)\right)^{\prime}\right)^{\prime}\right)^{\prime}+q_{0} t^{2} y(\lambda t)=0, \quad t \geq 1
$$

where $q_{0}>0$ and $\lambda \in(0,1]$. It is easy to verify that condition (2.10) is satisfied and by Theorem 1 , we conclude that any nonoscillatory solution of $\left(E_{x}\right)$ converges to zero as $t$ approaches infinity. 
By Theorem 2, we see that $\left(E_{x}\right)$ is oscillatory if

$q_{0}>6$

The same conclusion follows from Theorem 3 if $\lambda<1$ and

$$
q_{0} \ln \frac{1}{\lambda}>\frac{1}{\mathrm{e}}
$$

and from Theorem 4, if

$$
q_{0}>\frac{9}{2}
$$

Thus, Theorem 4 provides a stronger result than Theorem 2. Both theorems, however, do not depend on the value $\lambda$. In fact, Theorem 3 is more efficient for almost all values of $\lambda$, namely for $\lambda \in(0,0.9215)$.

\section{Acknowledgements}

The authors express their sincere gratitude to the editors for the careful reading of the original manuscript and useful comments, which helped to improve the presentation of the results and accentuate important details.

\section{Funding}

This research is supported by NNSF of P.R. China (Grant No. 61503171), CPSF (Grant No. 2015M582091), and NSF of Shandong Province (Grant No. ZR2016JL21). The research of the second and third authors is supported by the grant project KEGA 035TUKE-4/2017.

\section{Availability of data and materials}

Data sharing not applicable to this article as no datasets were generated or analysed during the current study.

\section{Competing interests}

The authors declare that they have no competing interests.

\section{Authors' contributions}

All four authors contributed equally to this work. They all read and approved the final version of the manuscript.

\section{Author details}

${ }^{1}$ Department of Engineering Mathematics, Faculty of Engineering, Cairo University, Giza, Egypt. ²Department of Mathematics and Theoretical Informatics, Faculty of Electrical Engineering and Informatics, Technical University of Košice Košice, Slovakia. ${ }^{3}$ School of Control Science and Engineering, Shandong University, Jinan, P.R. China.

\section{Publisher's Note}

Springer Nature remains neutral with regard to jurisdictional claims in published maps and institutional affiliations.

Received: 2 February 2019 Accepted: 11 March 2019 Published online: 20 March 2019

\section{References}

1. Agarwal, R.P., Grace, S.R.: The oscillation of higher-order differential equations with deviating arguments. Comput. Math. Appl. 38(3-4), 185-199 (1999)

2. Agarwal, R.P., Grace, S.R., Manojlovic, J.V.: Oscillation criteria for certain fourth order nonlinear functional differential equations. Math. Comput. Model. 44(1-2), 163-187 (2006)

3. Baculíková, B., Džurina, J., Graef, J.R.: On the oscillation of higher-order delay differential equations. J. Math. Sci. 187(4), 387-400 (2012)

4. Dennis, S.C.R., Walker, J.D.A.: Calculation of the steady flow past a sphere at low and moderate Reynolds numbers, J. Fluid Mech. 48(4), 771-789 (1971)

5. Džurina, J., Baculíková, B.: Oscillation of the even-order delay linear differential equation. Carpath. J. Math. 31(1), 69-76 (2015)

6. Džurina, J., Jadlovská, l.: Oscillation of third-order differential equations with noncanonical operators. Appl. Math. Comput. 336, 394-402 (2018)

7. Elias, U.: Oscillation Theory of Two-Term Differential Equations, vol. 396. Springer, Berlin (1997)

8. Esmailzadeh, E., Ghorashi, M.: Vibration analysis of beams traversed by uniform partially distributed moving masses. J. Sound Vib. 184(1), 9-17 (1995)

9. Grace, S.R., Agarwal, R.P., Graef, J.R.: Oscillation theorems for fourth order functional differential equations. J. Appl. Math. Comput. 30(1-2), 75-88 (2009) 
10. Grace, S.R., Bohner, M., Liu, A.: Oscillation criteria for fourth-order functional differential equations. Math. Slovaca 63(6), 1303-1320 (2013)

11. Graef, J.R., Grace, S.R., Tunç, E.: Oscillation of even-order advanced functional differential equations. Publ. Math. (Debr.) 93(3-4), 445-455 (2018)

12. Graef, J.R., Tunç, E.: Oscillation of fourth-order nonlinear dynamic equations on time scales. Panam. Math. J. 25(4), 16-34 (2015)

13. Kiguradze, I.T., Chanturia, T.A.: Asymptotic Properties of Solutions of Nonautonomous Ordinary Differential Equations. Mathematics and Its Applications (Soviet Series), vol. 89. Kluwer Academic, Dordrecht (1993). Translated from the 1985 Russian original

14. Ladde, G.S., Lakshmikantham, V., Zhang, B.G.: Oscillation Theory of Differential Equations with Deviating Arguments. Monographs and Textbooks in Pure and Applied Mathematics, vol. 110. Dekker, New York (1987)

15. Lega, J., Moloney, J.V., Newell, A.C.: Swift-Hohenberg equation for lasers. Phys. Rev. Lett. 73(22), 2978-2981 (1994)

16. Li, T., Rogovchenko, Yu.V.: Oscillation criteria for even-order neutral differential equations. Appl. Math. Lett. 61, 35-41 (2016)

17. Mahfoud, W.E.: Comparison theorems for delay differential equations. Pac. J. Math. 83(1), 187-197 (1979)

18. McKenna, P.J., Walter, W.: Nonlinear oscillations in a suspension bridge. Arch. Ration. Mech. Anal. 98(2), 167-177 (1987)

19. McKenna, P.J., Walter, W.: Travelling waves in a suspension bridge. SIAM J. Appl. Math. 50(3), 703-715 (1990)

20. Oğuztöreli, M.N., Stein, R.B.: An analysis of oscillations in neuro-muscular systems. J. Math. Biol. 2(2), 87-105 (1975)

21. Swanson, C.A.: Comparison and Oscillation Theory of Linear Differential Equations, vol. 48. Elsevier, Amsterdam (2000)

22. Trench, W.F.: Canonical forms and principal systems for general disconjugate equations. Trans. Am. Math. Soc. 189 319-327 (1974)

23. Truesdell, C.: Rational Mechanics. Academic Press, New York (1983)

24. Tunç, E.: Oscillation results for even order functional dynamic equations on time scales. Electron. J. Qual. Theory Differ. Equ. 2014, 27 (2014)

25. Zhang, C., Agarwal, R.P., Bohner, M., Li, T.: Oscillation of fourth-order delay dynamic equations. Sci. China Math. 58(1), 143-160 (2015)

26. Zhang, C., Agarwal, R.P., Li, T.: Oscillation and asymptotic behavior of higher-order delay differential equations with p-Laplacian like operators. J. Math. Anal. Appl. 409(2), 1093-1106 (2014)

\section{Submit your manuscript to a SpringerOpen ${ }^{\circ}$ journal and benefit from:}

- Convenient online submission

- Rigorous peer review

- Open access: articles freely available online

- High visibility within the field

- Retaining the copyright to your article

Submit your next manuscript at $\boldsymbol{~ s p r i n g e r o p e n . c o m ~}$ 